\title{
Pratiques
}

Linguistique, littérature, didactique

183-184| 2019

oralité, littératie

\section{Le livre numérique enrichi : quels enjeux de littératie en contexte pédagogique?}

\section{Nolwenn Tréhondart}

\section{(2) OpenEdition}

\section{Journals}

Édition électronique

URL : http://journals.openedition.org/pratiques/7732

DOI : $10.4000 /$ pratiques.7732

ISSN : 2425-2042

Éditeur

Centre de recherche sur les médiations (CREM)

Référence électronique

Nolwenn Tréhondart, «Le livre numérique enrichi : quels enjeux de littératie en contexte pédagogique? », Pratiques [En ligne], 183-184 | 2019, mis en ligne le 30 décembre 2019, consulté le 09 octobre 2020. URL : http://journals.openedition.org/pratiques/7732 ; DOI : https://doi.org/10.4000/ pratiques. 7732

Ce document a été généré automatiquement le 9 octobre 2020.

(C) Tous droits réservés 


\title{
Le livre numérique enrichi : quels enjeux de littératie en contexte pédagogique?
}

\author{
Nolwenn Tréhondart
}

\section{Introduction}

1 Si, en 2006, C. Belisle (p. 56) qualifiait le livre numérique de «technologie naissante et immature ", il est en 2020 un produit culturel commercialisable, soumis à des logiques d'industrialisation soutenues par les géants américains du Web : les Gafa' ${ }^{1}$. L'apparition de supports de lecture de plus en plus performants favorisant une lecture en mobilité (liseuses à encre électronique, tablettes numériques tactiles), la multiplication des librairies en ligne et des outils-logiciels de conception ont favorisé sa diffusion dans les usages en stabilisant peu à peu ses conditions socio-techniques et socio-économiques de production.

2 Dans ce contexte de développement des usages de la lecture numérique sur supports mobiles et tactiles, l'Éducation nationale développe depuis plusieurs années des politiques d'expérimentation. Au cycle 1, des écoles s'équipent de tablettes tactiles comme supports d'apprentissage ${ }^{2}$ tandis que le dispositif «Lycée 4.0 » de la région Grand Est vise à remplacer progressivement les manuels scolaires imprimés par des manuels numériques ${ }^{3}$.

3 Pour autant, le «livre numérique ", notamment sous sa forme "enrichie ", reste un objet protéiforme, mal identifié. En quête d'une légitimité culturelle, il ne cesse, d'une part, d'emprunter aux codes et aux canons de l'imprimé, mimant les formes graphiques du livre jusque dans le design de ses interfaces. D'autre part, en tant que média informatisé, il préfigure de nouveaux cadres d'interprétation du « texte ${ }^{4}$ » en raison de ses spécificités multimodales qui s'appuient sur une pluralité de ressources sémiotiques - texte, image, son et vidéo. 
4 À l'heure de l'hybridation des supports de lecture (livres, tablettes, ordinateurs, smartphones...), cette tension entre rupture et continuité nous semble former un cadre heuristique intéressant pour questionner les représentations liées aux évolutions des pratiques sociales de l'écrit. Nous nous proposons dans cet article d'examiner la manière dont l'objet "livre numérique enrichi", comme dispositif symbolique et technique, à travers ses formes graphiques et les représentations qu'il suscite, pourrait constituer une porte d'entrée pertinente dans l'apprentissage d'une «littératie médiatique multimodale » en contexte numérique (Boutin, Lacelle \& Lebrun, 2012).

5 Nous délimiterons, dans un premier temps, notre objet d'étude - le «livre numérique enrichie »- au regard de sa spécificité "multimodale» qui conjugue sur un même support une pluralité de ressources sémiotiques (textuelles, sonores, visuelles, gestuelles...). Puis, en nous appuyant sur des entretiens que nous avons menés entre 2011 et 2016 auprès de concepteurs de livres numériques enrichis dans les genres de la fiction et du livre d'art, nous chercherons à mettre en relief le faisceau de représentations paradoxales que les concepteurs mobilisent lors du processus de création, ainsi que les enjeux de pouvoir liés à son économie scripturaire fortement dépendante des stratégies d'industrialisation des Gafa. Enfin, dans un contexte où les enseignants sont invités à élargir leurs approches de l'apprentissage de l'écriture et de la lecture à l'aune des pratiques numériques, nous reviendrons sur certains enjeux d'apprentissage de la « littératie médiatique multimodale », en proposant d'en élargir le périmètre par une lecture critique des dispositifs de lecture et de publication.

\section{Le livre numérique enrichi, support d'apprentissage d'une " littératie médiatique multimodale »}

\subsection{Précisions terminologiques : " homothétiques ", « enrichis ", " augmentés "}

C. Bélisle (2006, p. 50-51) propose de définir le livre numérique comme :

un objet composé d'au moins quatre éléments : un support physique (une tablette électronique), un logiciel d'exploitation, un logiciel de lecture et des fichiers correspondant à chacune des œuvres copiées dans la mémoire du support.

7 Cette description met l'accent sur le fait que le livre numérique n'est pas juste un «fichier » numérique mais le résultat de l'actualisation de ce fichier à l'intérieur d'un dispositif technique qui oriente en amont les conditions de production et de réception : ainsi, un livre au format $\mathrm{ePub}^{5} \mathrm{~s}^{\prime}$ affichera différemment à l'écran selon le logiciel ou le support de lecture utilisés (taille de la police, format de la page...).

Actuellement, les usages terminologiques tendent à répartir les livres numériques en trois grandes catégories : « homothétiques », « enrichis » et « augmentés ».

9 Le livre numérique "homothétique " correspond à la transposition numérique d'un ouvrage préalablement imprimé : il s'agit d'une « simple translation du contenu, sans mise en forme ajoutée » (Audet, 2015), dont « l'usage reste très proche de celui du livre papier, à la fois dans sa présentation graphique et dans son ergonomie " (Lekehal, Maurin \& Prost, 2013, p. 36).

Le livre numérique « enrichi », s'il reproduit également des similitudes avec l'imprimé, s'ouvre en parallèle aux spécificités créatives de l'écriture numérique, en exploitant 
son potentiel hypertextuel, animé et manipulable (Saemmer, 2015). B. Prost le définit comme :

La prolongation naturelle du livre homothétique: il s'agit de profiter des possibilités offertes par l'informatique et d'explorer de nouvelles voies de création rendues possibles par la dématérialisation, le multimédia et l'Internet. L'orientation la plus intuitive pour enrichir le livre consiste à introduire un contenu additionnel au livre d'origine. Avec le numérique, l'auteur n'est plus limité par un volume de pages prévisionnel : il peut ajouter du texte sans limites, et surtout il peut faire appel à des médias temporels comme la vidéo ou le son intégrés au sein du texte, alors que dans le monde du papier, la seule possibilité reste l'adjonction d'un support optique (CD ou DVD) ou l'indication de liens Internet (non cliquables évidemment) à l'intérieur du texte. (Lekehal, Maurin \& Prost, 2013, p. 38).

Enfin, le livre numérique "augmenté » se distingue du livre numérique enrichi, en proposant des formes de lecture transmédiatiques, reposant sur l'association entre un livre papier et une application de réalité augmentée (Rio, 2014).

\subsection{De l'enrichissement...}

Pour autant, l'expression « livre numérique enrichi » ne fait pas l'objet d'un consensus. Pour A. Guilet (2015), l'emploi du mot «livre » y participerait d'un abus de langage, d'une rhétorique commerciale, cherchant «à intégrer à tout prix les œuvres numériques dans une tradition livresque "; si l'on définit le livre du point de vue de sa matérialité comme un "assemblage d'un assez grand nombre de feuilles [...], portant des signes destinés à être lus » (Le Petit Robert, 2004, p. 1503), ces contenus littéraires et culturels téléchargeables sur tablette ou liseuse ne sont pas au sens propre des "livres"; dès lors, la chercheuse propose de parler plutôt d'« œuvres hypermédiatiques pour écrans tactiles ", pour penser leurs conditions d'existence en dehors du support «livre» et resituer la réflexion dans l'histoire plus longue de la littérature hypertextuelle et hypermédiatique (Bouchardon, 2014). De son côté, M. Jajah (2010) estime que ce n'est pas tant la forme « livre » que cette expression désigne, mais plutôt une tension entre "une façon de concevoir et de penser propre au livre » et "une façon de faire qui pense non plus en vue d'une réalisation papier, mais directement numérique. » Certes, les « enrichissements " propres au livre numérique ont été définis par un décret en 2011 qui précise qu'il s'agit des "variations typographiques et de composition, modalités d'accès aux illustrations et au texte [...], ajouts de textes ou de données relevant de genres différents, notamment sons, musiques, images animées ou fixes, limités en nombre et en importance, complémentaires du livre et destinés à en faciliter la compréhension $»^{6}$. Pour autant, cette idée d'enrichissement laisse, d'une part, supposer l'existence d'un texte premier, originel, auquel auraient été «ajoutés » d'autres médias, mais renvoie aussi à l'idée de valeur ajoutée, comme si l'objet second «enrichi » gagnait en profondeur, en valeur, par l'ajout de ressources numériques (interactivité, agrandissement...).

C'est précisément en raison des débats qu'elle suscite que l'expression "livre numérique enrichi » nous semble intéressante : elle illustre, selon nous, le paradoxe entre l'idée d'un objet toujours jugé "à l'aune de la formule maîtresse du livre " (Robert, 2009, p. 216), et la possibilité d'une échappée créative grâce aux spécificités hypermédiatiques de l'écriture numérique ouvrant la voie à l'expérimentation de nouvelles formes de littérarité. 


\section{3 ... à la multimodalité en contexte scolaire}

14 Le terme d'enrichissement est également questionné par A. Laborderie, C. Jeantet et P. Hellégouarc'h (2018) au prisme du concept de « multimodalité », selon une approche qui s’inscrit dans le champ de recherche québécois sur la «littératie médiatique multimodale » (Boutin, Lacelle \& Lebrun, 2012). Celle-ci est définie comme :

la capacité d'une personne à mobiliser adéquatement, en contexte communicationnel synchrone ou asynchrone, les ressources et les compétences sémiotiques modales (ex.: mode linguistique seul) et multimodales (ex.: combinaison des modes linguistique, visuel et sonore) les plus appropriées à la situation et au support de communication (traditionnel ou numérique), à l'occasion de la réception (décodage, compréhension, interprétation et évaluation) ou de la production (élaboration, création, diffusion) de tout type de message. (Boutin, Lacelle \& Lebrun, 2017)

15 Cette définition, fondée sur les habiletés (décoder, comprendre, interpréter, évaluer, élaborer, créer, diffuser), ouvre, selon ces chercheurs, de nouvelles perspectives en termes d'apprentissage. Elle souhaite dépasser une "vision verbo-centrée " de la littératie, d'une part, en la colorant des formes de communication que supposent les médias, et d'autre part, en adoptant la perspective en sémiotique sociale développée par G. Kress (2010). Celle-ci étudie la manière dont la production de sens mobilise en contexte multimodal différentes ressources sémiotiques, rattachées à un éventail de pratiques sociales et de représentations. L'image, l'écriture, la gestuelle, le regard, la parole, sont à envisager comme des « ressources sémiotiques " socialement, historiquement et culturellement construites, dont certaines seront privilégiées à une époque et dans un contexte donnés.

Pour Laborderie, Jeantet, \& Hellegouarc'h (2018), la dimension multimodale du livre numérique enrichi permet de multiplier les perspectives et modes d'entrée dans le texte: elle ouvrirait, en effet, sur « un espace textuel et sensoriel renouvelant profondément l'expérience visuelle et cognitive de la lecture », à la fois en termes de signification mais aussi d'attention et de perception. Le livre numérique enrichi semble, dès lors, un support idéal dans l'apprentissage d'une littératie médiatique multimodale tant ses formes éditoriales convoquent une pluralité de ressources sémiotiques (textuelles, visuelles, sonores, gestuelles) qui favorisent l'exploration de diverses manières de produire du sens. Au cycle 3 , le programme de français recommande ainsi d'initier les apprenants à la lecture «de documents numériques (documents avec des liens hypertextes, documents associant texte, images - fixes ou animées -, $\left.\operatorname{son}^{7}\right) »$. Le programme du cycle 4 invite à les familiariser avec les " potentialités et usages des nouveaux supports de l'écriture » en les entraînant à la composition "d'écrits créatifs sur différents supports ${ }^{8}$ " (blogs, écrits collaboratifs en ligne...). Pour autant, ces incitations à sensibiliser les apprenants aux spécificités de l'écriture numérique entrent en tension avec la montée des inquiétudes de la communauté éducative face à l'utilisation excessive des dispositifs numériques, soupçonnés de susciter des comportements d'inattention chez les apprenants : dans quelle mesure, par exemple, l'insertion d'animations sonores ou visuelles facilite-t-elle la compréhension en concrétisant le langage ou, au contraire, distraie-t-elle du fil conducteur d'une histoire? Ces interrogations sont intéressantes en ce qu'elles viennent révéler certains enjeux d'appropriation en contexte numérique, et illustrent le conflit entre une lecture d'« hyperattention », décrite par K. Hayles (2017), comme 
" un mode de cognition qui tolère très mal l'ennui, alterne avec souplesse entre différents flux d'information et préfère un haut degré de stimulation » - et une lecture soutenue dont le " modèle cognitif, [...] préfère un flux d'information unique, se concentre sur un objet culturel unique pour une période relativement longue et a une grande tolérance à l'ennui ».

Ensuite, nous proposons de confronter ces interrogations sur la lecture numérique en contexte multimodal aux résultats d'une enquête que nous avons conduite entre 2011 et 2016 auprès d'un groupe de trente concepteurs de livres numériques enrichis (chefs de projets éditoriaux, auteurs auto-édités et designers ${ }^{9}$ ) dans les genres de la fiction et du livre d'art numériques (Tréhondart, 2016). À partir d'entretiens semi-directifs, nous avons cherché à mieux comprendre les représentations du "lire " qu'ils mobilisent pour imaginer et concevoir le design éditorial de leurs productions, et les questions qu'ils se posent dans le choix et l'hybridation de ressources sémiotiques hétérogènes.

\section{Le livre numérique enrichi, un objet pris dans un faisceau de représentations paradoxales}

Avec cette enquête, nous proposons de dessiner les contours de l'objet «livre numérique enrichi » à partir du point de vue de ses concepteurs, selon l'idée que :

Le processus de production est façonné de bout en bout par des idées: un savoir usuel concernant les procédures courantes de production, des compétences techniques historiquement définies, des idéologies professionnelles, des définitions et des suppositions, des hypothèses sur le public, et ainsi de suite... (Hall, 1994 [1973], p. 30).

$19 \mathrm{Si}$, dans le cas du livre numérique, le savoir usuel peut s'appuyer sur des traditions de métier et des études sur les pratiques de lecture numérique ou les usages de la tablette, les concepteurs avancent aussi des hypothèses sur les modes d'appropriation des lecteurs (Akrich, 2010). Celles-ci s'appuient sur un éventail de valeurs, de normes, de représentations, dont nous avons cherché à faire ressortir le caractère paradoxal.

\subsection{Filiations livresques : entre clôture et ouverture}

Pour Yves Jeanneret (2007, p. 85), le livre numérique incarne « l'espoir d'avoir tout à la fois: la modernité d'un concept révolutionnaire et la pérennité d'un objet patrimonial ». Sa proximité avec les formes du livre imprimé reflète notre attachement culturel profond pour l'objet matériel livre et son héritage - certains effets de métaphore se retrouvant jusque dans les "pages qui se tournent» des interfaces matérielles et logicielles des tablettes numériques. Durant les entretiens, les concepteurs insistent sur leur volonté de s'inscrire dans cet héritage. Le livre numérique enrichi s'entend comme la «remédiatisation " (Bolter \& Grusin, 2000) à l'écran d'une forme culturelle préexistante, dont ils entendent perpétuer l'héritage en interrogeant "son historique centenaire " (A28) ou en la "réinterprétant à l'écran " (A30). À ce titre, les repères graphiques liés au livre restent essentiels et se matérialisent dans les formes-modèles suivantes :

- la couverture - présentée comme un "sas de décompression » qui appelle d'emblée à un comportement de lecteur ;

- le sommaire - qui représente le volume global d'informations ; 
- la page - marqueur culturel central ;

- l'« enveloppe » liée au fichier téléchargeable du livre numérique qui permet d'actualiser les contenus à l'intérieur d'une forme circonscrite.

21 Cette idée de clôture semble d'autant plus nécessaire quand la lecture s'effectue sur le support "tablette», dont la polyvalence tend, selon les concepteurs, à détourner l'attention du lecteur vers une multitude d'activités ${ }^{10}$. Il s'agit de penser le livre numérique enrichi «non pas comme un site illimité, mais comme un objet, une pierre posée sur le Web », confie l'un des concepteurs (A18). Ces propos résonnent avec ceux de M. Melot (2006, p. 78) qui, dans un entretien avec J. Goody, revient sur cette idée de finitude matérielle : en donnant une limite à la pensée, la forme circonscrite du livre serait au fondement de son autorité. Ainsi chez les concepteurs interrogés, l'idée d'une clôture "numérique » qui délimiterait le texte dans un espace d'appropriation donné, tout en entrant en tension avec les possibilités d'ouverture du texte numérique, semble s'imposer : ces derniers estiment d'ailleurs qu'il y a avec le numérique une « possibilité infinie d'expressions» (A12) quand avec «le livre papier, il n'y a pas 15000 possibilités » (A32).

Exemple 1 : Capture écran d'une des pages du livre numérique enrichi Beauvoir, l'enquête par Irène Frain (coédition Storylab-Lafon, 2014)

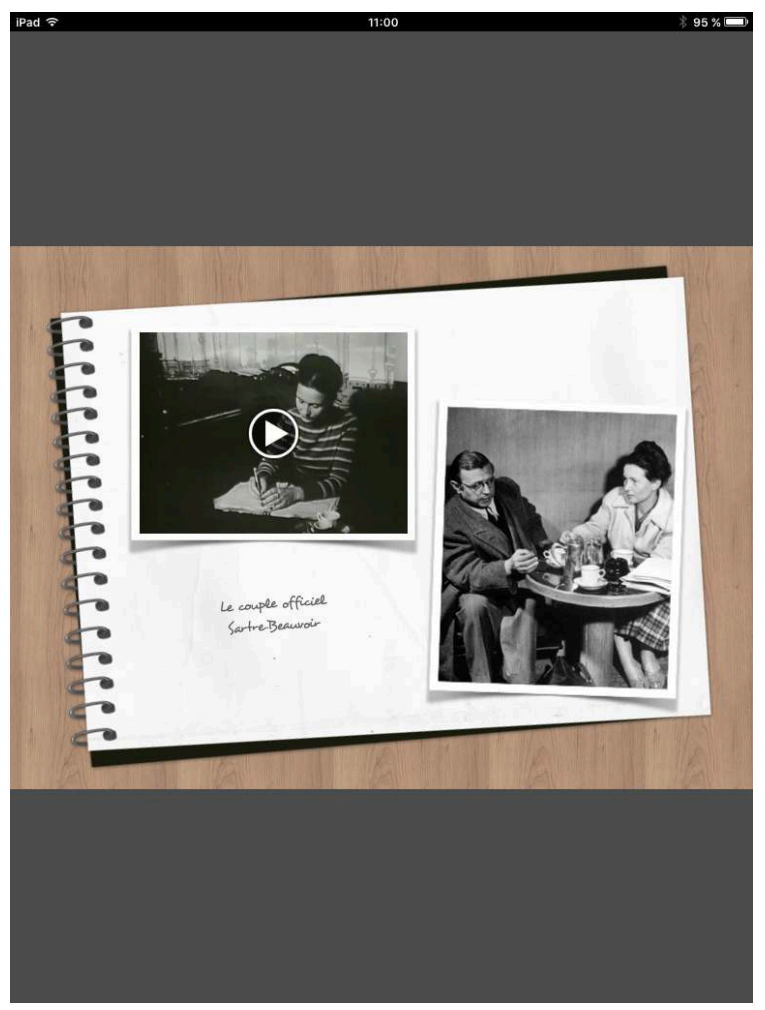


Exemple 2: Capture écran du chemin de fer du livre numérique enrichi Le Horla (édition : L'Apprimerie, 2015)

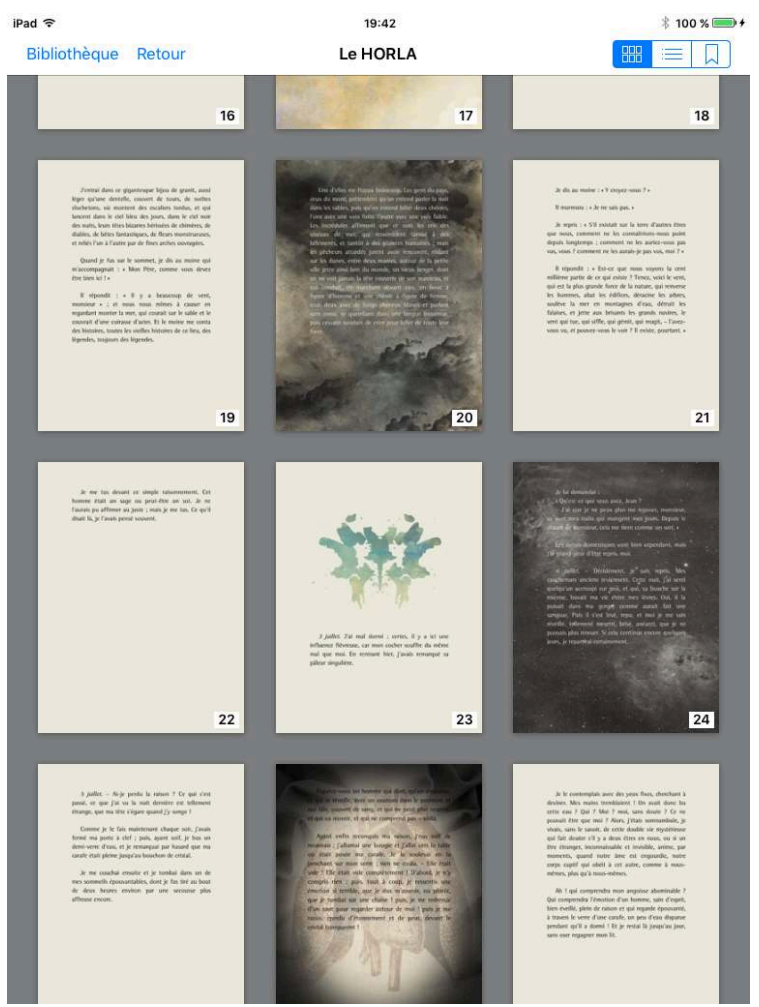

Exemple 3 : Capture écran de la « couverture animée » du livre numérique enrichi Le Horla (édition : L'Apprimerie, 2015)

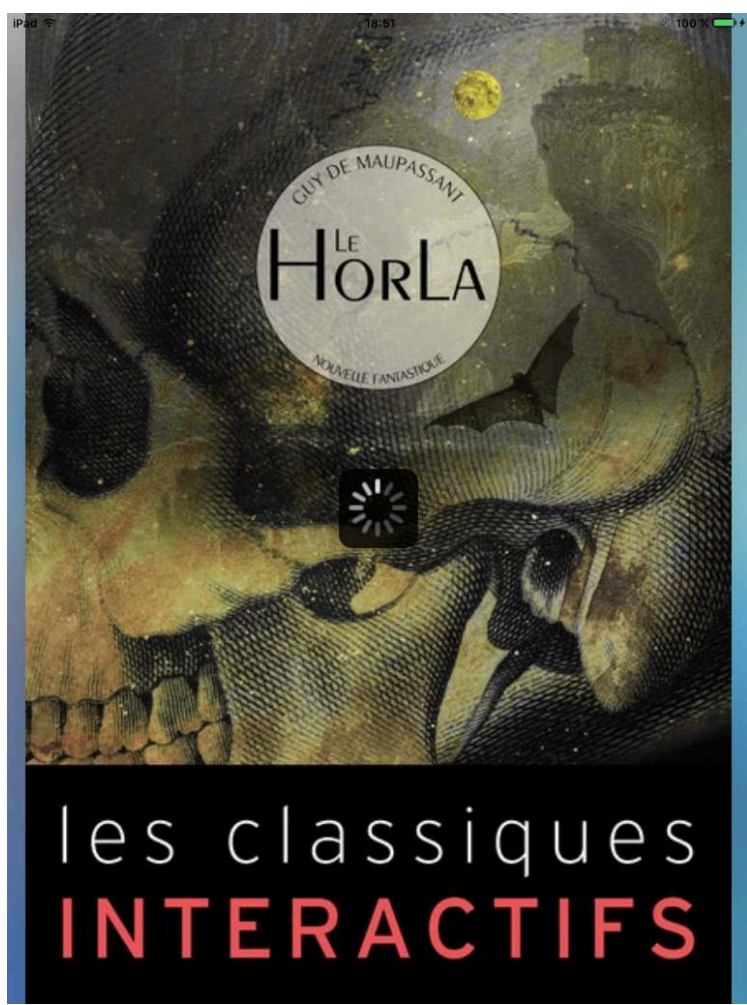




\section{Lecture multimodale : recentrage ou dispersion de l'attention?}

Objet multimodal, le livre numérique enrichi convoque des ressources sémiotiques hybrides. Ainsi, dans le catalogue d'exposition numérique Edward Hopper, d'une fenêtre à l'autre ${ }^{11}$, plusieurs œuvres du peintre sont introduites par des séquences animées, conçues comme des assemblages sonores et visuels d'œuvres et de citations mises en perspective par la voix du commissaire d'exposition D. Ottinger. Beaucoup de livres numériques pour la jeunesse sollicitent l'ouïe du lecteur - narration contée -, ou des gestes de manipulation qui l'amènent à déplacer des objets à l'écran ou à les toucher pour déclencher des animations.

Exemple 4 : Capture écran d'une des « séquences animées " proposées par l'application Edward Hopper, d'une fenêtre à l'autre (Édition : Réunion des musées nationaux, 2012)
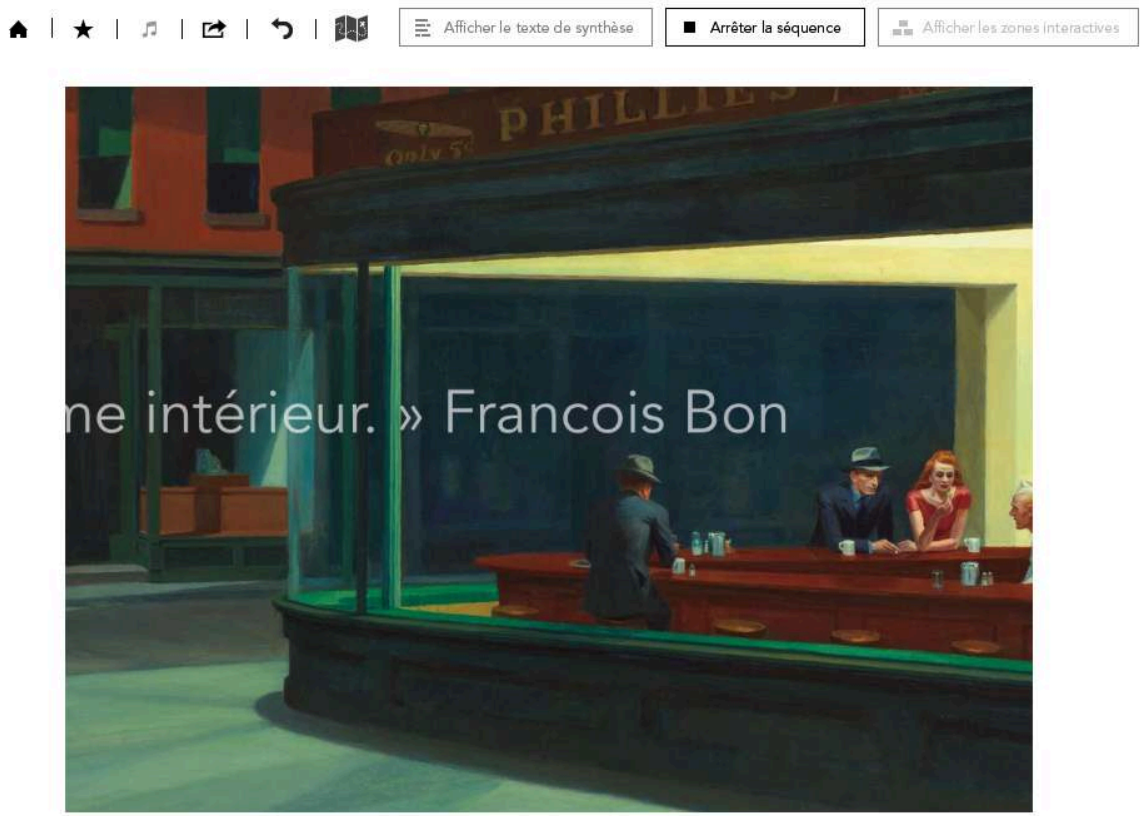
Exemple 5 : Capture écran d'une des pages du livre numérique enrichi Le Horla (édition :

L'Apprimerie, 2015). Quand le lecteur touche un arbre, celui-ci s'écarte en dévoilant un pan du texte.

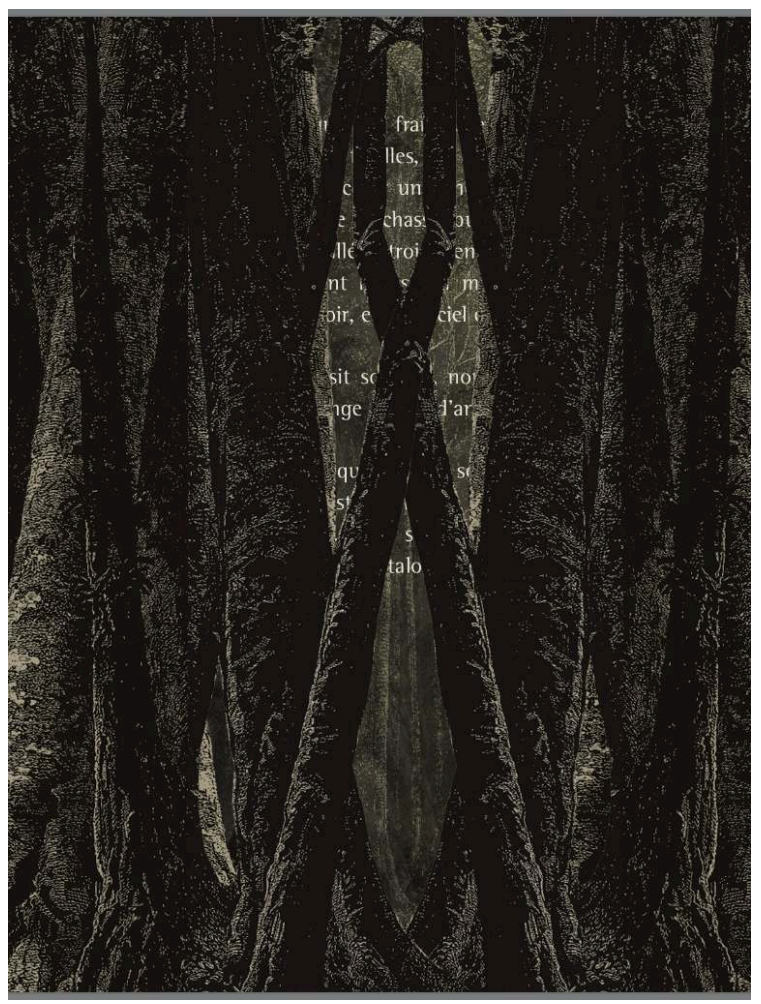

Exemple 6 : Capture écran d'une des pages du livre numérique enrichi Le Horla (édition : L'Apprimerie, 2015). Le texte disparaît progressivement à l'écran.

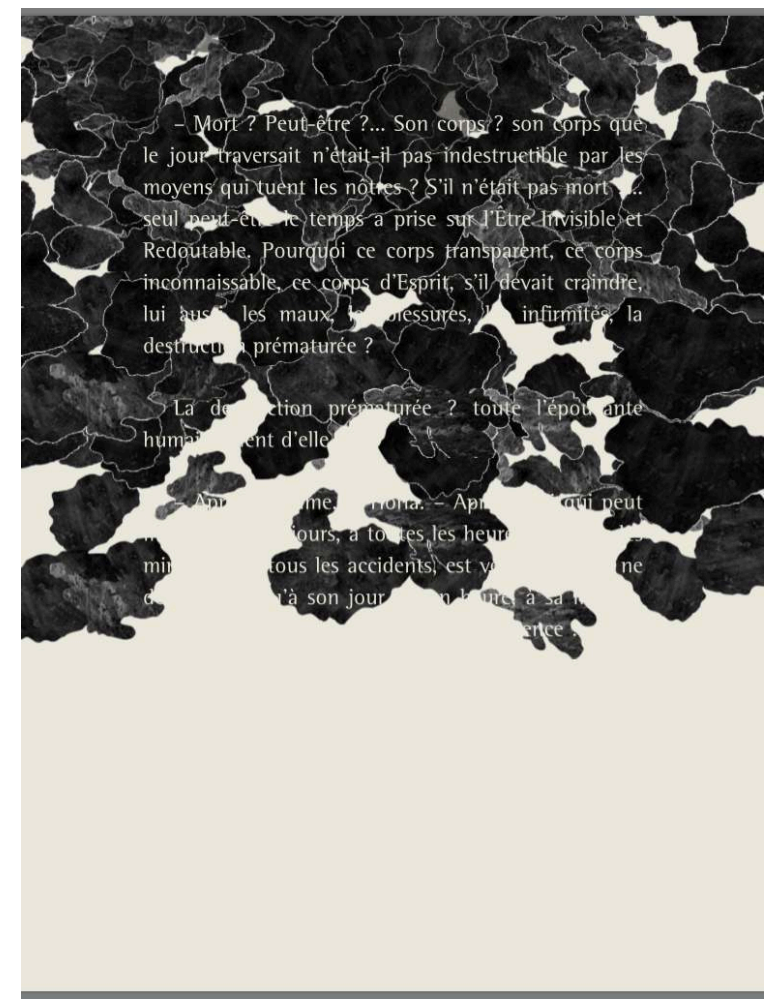



paradoxaux. Ils déclarent vouloir expérimenter la richesse multimodale du texte numérique, tout en limitant le nombre d'interactions pour éviter que les modes de lecture visuels, sonores ou gestuels prennent le pas sur le mode textuel (le déchiffrage des signes écrits). Soupçonnée d'inciter à des comportements ludiques, l'interactivité ${ }^{12}$ est ainsi soupçonnée d'engendrer une certaine fascination envers le dispositif numérique et le spectacle des formes animées :

« Est-ce que je lis l'œuvre que j'ai lancée ou est-ce que je la joue ?» (A9)

"C'est plus des livres, c'est des jeux, l'enfant passe son temps à cliquer sur des

formes. » (A8)

«Les gens ne lisent pas l'histoire, ils profitent juste des interactions. » (A11)

De telles craintes ne sont pas nouvelles : A. Saemmer (2011, p. 66) note que, sur support numérique, le «rapport entre observation, écoute, lecture et interaction [...] peut mettre en danger le texte comme signifiant potentiel ». N. Xanthos et B. Gervais (1999) évoquent, eux aussi, le risque d'épuisement et d'enfermement dans des formes d'exploration ludiques sur supports numériques. À ce titre, les concepteurs semblent défendre une conception "intensive » de la lecture sur support numérique, à rebours d'une conception "extensive", s'inscrivant, selon B. Gervais $(1998$, p. 8) dans un contexte de consommation rapide des biens culturels. Leurs dilemmes de concepteurs font aussi écho aux propos d'A. Giffard (2010) qui décèle, en régime numérique, un conflit entre une "attention orientée texte" héritée de la culture classique et une « attention orientée médium » caractérisée par d'incessantes relances médiatiques.

\section{L'hypertexte, liberté ou désorientation du lecteur?}

La lecture numérique est régulièrement qualifiée de lecture de survol, voire de " pseudo-lecture » (Giffard, 2010), qui alimenterait des pratiques impatientes, agitées de clics et de scrolls. C. Vandendorpe $(1999$, p. 228) estime ainsi que la " consultation de l'hypertexte est placée sous le signe de l'immédiateté et de l'urgence » : le lecteur serait tellement excité à l'idée de dévoiler un hyperlien qu'«il voudrait être arrivé à destination avant même que d'avoir commencé à lire ». À contrario, le champ de la littérature numérique (Bouchardon, 2014) s'est, de longue date, emparé de l'hypertexte, pour créer des "hyperfictions" proposant au lecteur de jouer un rôle actif dans la construction de l'histoire - elon le concept de wreader (Landow, 1992) («lecteurauteur »). Si les précurseurs de la littérature numérique ont affiché leur volonté de remettre en cause les modèles intériorisés de la lecture linéaire ainsi, qu'en pensent les concepteurs interrogés?

Beaucoup perçoivent l'hyperlien comme un outil de démocratisation de la lecture, une liberté accordée au lecteur, qui facilite l'accès aux contenus, en l'aidant à «construire le chemin de fer de l'ouvrage " (A15). Il est cependant aussi critiqué en raison de sa dimension "haptique» (Mangen, 2008), qui introduit un processus de «lecture gestualisée » (Saemmer, 2015) engageant le corps de manière plus consciente qu'avec le support du livre imprimé : sur écran, le lecteur doit en effet cliquer sur des hyperliens ou toucher des icônes pour progresser et s'approprier le sens, au lieu de simplement «tourner les pages». De nombreux concepteurs abordent l'hyperlien comme un outil " efficace", permettant de combler un besoin d'information, mais ne souhaitent pas s'en emparer comme un outil au service d'une pratique narrative, estimant que les 
hyperfictions expérimentales ont découragé les lecteurs par leur structure labyrinthique.

«On n'a pas réalisé à quel point cela pouvait désorienter le fait de suivre le fil conducteur en cliquant sur le lien.» (A19)

«Les gens vont directement cliquer sur les mots au lieu de lire ce qui précède. » (A11).

«Le principe de mots cliquables, touchables qu'on a mis en place, perturbe la lecture, casse l'immersion. » (A11)

\section{La captation des pratiques des pratiques de création}

S'il questionne les représentations liées à la lecture, le livre numérique s'inscrit aussi dans des jeux de pouvoir portés par les grands acteurs du numérique. Fruit d'une « énonciation éditoriale » collective (Jeanneret \& Souchier, 2005), son design n'est pas seulement décidé par son concepteur, mais repose sur les prescriptions techniques et esthétiques émises par les producteurs de technologies et les constructeurs de dispositifs. Au détour des entretiens, il apparaît que l'élément déclencheur ayant motivé la décision de se lancer dans la création et la commercialisation de livres numériques enrichis a été le lancement de la tablette iPad par la société Apple en 2010. Sa gestuelle tactile, son format vertical, sa nomadicité, sa simplicité apparente d'utilisation, mais aussi son design épuré et élégant raviveraient, selon les concepteurs, des postures familières de lecture qui «réconcilieraient le littéraire avec l'informatique » (A12). Le format «applicatif » iOS d'Apple est vanté pour la liberté de design qu'il offre: son univers clos et la discrétisation des signes informatiques permettraient d'effectuer "un choix poussé dans la mise en pages» (A17), de " construire sa planète d'auteur " (A20). Reprenant les allants de soi de la transparence et de l'immédiateté des dispositifs numériques, beaucoup de concepteurs valorisent l'interface tactile de la tablette pour sa dimension «intuitive » et "naturelle », qui favoriserait l'immersion dans les contenus.

Pour autant, si Apple crée un milieu favorable à la création et à la diffusion, les concepteurs dénoncent en parallèle le cadrage de leurs pratiques dans des schémas technologiques propriétaires. Ils comparent le système Apple à une " prison dorée », en expliquant comment ils doivent aussi composer avec les contraintes de l'environnement de production: ils explicitent la manière dont les injonctions à la gratuité en vigueur sur la plateforme de vente de l'App Store constituent une entrave au développement de leur modèle économique; les formes-modèles imposées par l'outil-logiciel de lecture iBooks viennent freiner leur élan créatif, sans compter les phénomènes de censure sur des contenus jugés «inappropriés » par les politiques éditoriales des plateformes. Oscillant entre adhésion et résistance à ces tentatives de cadrage de leurs pratiques, ils ne se montrent pas dupes des stratégies de "capture " des métiers et de l'attention que mettent en place les Gafa dans l'industrialisation du processus de production et de commercialisation du livre numérique enrichi.

Cette enquête nous a permis de mettre en relief certains champs de tension propres au «livre numérique enrichi», entre héritage des conventions livresques, expérimentations hésitant à déporter le lecteur aux "limites de ses habitudes" (Gervais, 1998) et enjeux de pouvoir liés au rôle joué par les constructeurs de supports. Malgré leur caractère ambivalent, paradoxal, nous faisons l'hypothèse que ces tiraillements peuvent constituer une opportunité dans l'apprentissage d'une littératie médiatique multimodale, en offrant un cadre de pensée heuristique. Dans une dernière 
partie, nous proposons ainsi deux pistes qui seraient - selon nous- intéressantes à explorer en contexte scolaire : le travail sur les processus d'interprétation en contexte multimodal ; la sensibilisation à la lecture critique des supports et logiciels de lecture.

\section{Quels enjeux de littératie en contexte pédagogique?}

\subsection{Prendre en considération les processus d'interprétation plutôt que les scénarios d'utilisation}

Sur le terrain, les pratiques pédagogiques semblent se focaliser sur la création de livres numériques via des logiciels de conception faciles à prendre en main plutôt que sur le processus interprétatif. En nous resituant dans la perspective de l'apprentissage d'une "littératie médiatique multimodale", il nous semblerait pourtant intéressant de prendre appui sur le faisceau de représentations paradoxales qui entourent l'objet « livre numérique enrichi »- entre emprunts et ruptures, création et standardisation, clôture et ouverture - pour explorer en classe les représentations liées à l'écrit et à la lecture. Les formes du livre numérique enrichi qui revendiquent leur inscription dans l'héritage des conventions imprimés tout en explorant le potentiel poétique et narratif de l'hypertexte, de l'animation et de l'hybridation entre texte, image fixe, son et vidéo, peuvent s'avérer d'un abord plus facile que des œuvres émanant du champ expérimental - et plus radical - de la littérature numérique ${ }^{13}$. Le questionnement sur le processus interprétatif à partir de l'hybridation de différentes ressources sémiotiques nous semble de nature à favoriser la confrontation des points de vue et valeurs qui guident les pratiques des apprenants, tout en favorisant un regard réflexif.

31 De telles expérimentations peuvent être menées dès la maternelle, dès les premiers rapports à l'écrit. Dans le cadre de son mémoire de Master 2 (Pini, 2019), l'une de nos étudiantes a mené une expérimentation en première section, qui confrontait les enfants à deux situations de lecture : un premier groupe lisait un album imprimé pour la jeunesse ; le second, son adaptation sous forme d'album numérique enrichi. L'objectif était d'observer l'évolution des pratiques de classe liées à la différence de nature des albums, mais aussi de faire évoluer les représentations de la tablette comme dispositif purement ludique. Pendant l'expérience, il a été intéressant de constater le changement de posture de l'enseignante. En effet, en maternelle, la pratique du livre débute avec la manipulation de l'objet album ainsi qu'avec l'oralisation des pratiques de lecture : le texte est commenté, chanté, performé par l'enseignant. Comme le souligne F. Grosmann (1996, p. 88), l'album pour enfants a " pour caractéristique de ne pouvoir se lire (au sens propre) qu'au cours d'une interaction établie entre l'adulte (ou l'enfant lecteur) et un public formé de "non-lecteurs" ». Si, dans la première situation de lecture, l'enseignante pratiquait une "lecture magistrale» en retournant régulièrement l'album pour en montrer les images aux enfants, dans le second cas, elle s'est placée en situation d'observatrice, les laissant manipuler en toute autonomie. À l'issue de l'expérimentation, les enfants ont déclaré avoir tout particulièrement apprécié de pouvoir manipuler à leur guise l'album numérique, notamment parce qu'ils pouvaient, tout en "feuilletant ", écouter à volonté la voix du narrateur médiée par le dispositif. Néanmoins, certaines hybridations sémiotiques - entre les modes sonores et visuels notamment - ont semblé résister à l'interprétation, les enfants peinant à comprendre que « derrière un son peut aussi se cacher un sentiment, une émotion, une 
information" (Pini, 2019, p. 47). L'on voit bien, à travers cette expérimentation, comment la mise en place d'un travail d'interprétation autour du livre numérique enrichi vient questionner des pratiques de classe établies et est susceptible d'en transformer les rituels, notamment ceux liées à l'oralisation de la lecture de l'album. Il serait intéressant à terme de travailler sur les formes de littératie engendrées par l'hybridation des supports papier et numérique.

\subsection{Lecture critique des dispositifs de lecture et de publication}

Par ailleurs, les entretiens avec les concepteurs ont permis de montrer que les formes sémiotiques du livre numérique enrichi sont le produit de jeux de pouvoir, de compromis et de négociations entre acteurs (concepteurs-éditeurs-designers-auteurs, constructeurs de dispositifs) et dispositifs (outils-logiciels, tablettes, smartphones...). Or, le rôle du support et de son environnement (technologique, économique) dans l'orientation des pratiques de production et de réception est encore peu pris en compte dans les pratiques pédagogiques : la tablette reste appréhendée comme un canal neutre de diffusion de contenus ou auréolée des discours sur le numérique qui la valorisent comme vecteur de motivation et de pratiques pédagogiques « innovantes ». En ce sens, il paraîtrait pertinent d'étendre la définition de la «littératie médiatique multimodale» (Boutin, Lacelle \& Lebrun, 2012) à la compréhension des rapports de pouvoir qui se trament au sein des dispositifs numériques de lecture et de publication. Pour ce faire, il est possible de s'appuyer sur le concept d'« architexte " proposé par Y. Jeanneret et E. Souchier (2005) qui nomment ainsi les formes programmées et automatisées qui régissent les conditions de l'écriture à l'écran :

Nous nommons architextes (de archè, origine et commandement), les outils qui permettent l'existence de l'écrit à l'écran et qui, non contents de représenter la structure du texte, en commandent l'exécution et la réalisation. Autrement dit, le texte nait de l'architexte qui en balise l'écriture. (Jeanneret, Le Marec \& Souchier, 2003, p. 23-24)

La création de livres numériques enrichis avec les élèves gagnerait donc à aller au-delà du seul décodage des signes expérimentables à la surface de l'écran, en s'accompagnant d'une sensibilisation critique au rôle des dispositifs d'actualisation (ordinateur, smartphone) et des outils-logiciels de production. Comment les constructeurs de logiciels et de plateformes d'écriture organisent-ils les pratiques d'écriture selon des intérêts stratégiques spécifiques? Pour initier ce rapport critique à la création, les élèves pourraient, par exemple, analyser le design de plusieurs livres numériques enrichis, et dévoiler collectivement leurs représentations face à cet objet souvent inconnu. Ce travail permettrait de les sensibiliser aux lectures préférentielles modélisées par les outils-logiciels tels que iBooks Author ou Book Creator et les formesmodèles qu'ils imposent. Ici, l'enjeu est de prendre conscience à la fois du caractère fortement contraint des formes automatisées de l'écriture en régime numérique, mais aussi du champ des possibles ouverts par les procédés rhétoriques du texte animé et manipulable.

\section{Conclusion}

34 À travers cet article, nous avons souhaité montrer combien le «livre numérique enrichi», objet culturel encore mal identifié, est pris dans un faisceau de 
représentations contradictoires. Nous nous sommes appuyés sur une enquête conduite auprès de concepteurs dans les genres de la fiction et du livre d'art pour illustrer certains de ces champs de tension. D'un côté, les formes éditoriales du livre numérique enrichi témoignent de la prégnance de l'imaginaire livresque, dans leur emprunt aux normes et conventions héritées de l'écrit et de l'imprimé (imaginaires du sommaire et de la page remédiatisés à l'écran, clôture narrative, habitus gestuels liés au «feuilletage »...). De l'autre, les propos des concepteurs s'inscrivent dans un désir de création porté par le potentiel de l'écriture hypermédiatique (interactivité, hypertexte...) et un souhait de standardisation dans un contexte où se développent de nouvelles formes de domination scripturaire liées à l'industrialisation numérique de l'économie.

Comment dès lors aborder les enjeux liés à l'apprentissage de la littératie médiatique multimodale? Quelles formes de design pédagogiques inventer pour dépasser la simple familiarisation des apprenants avec les outils-logiciels de production? Il nous semble que c'est justement en raison de ses multiples paradoxes que le livre numérique enrichi peut servir de point d'appui à l'apprentissage d'une littératie en contexte numérique. Un tel objectif nécessite de développer en classe une culture de l'interprétation, qui prend en considération les représentations des apprenants, afin de les amener à questionner leurs regards et points de vue sur leurs pratiques. Cela nécessite également, selon nous, d'élargir le périmètre de la définition de la littératie médiatique multimodale à la lecture critique des dispositifs de lecture et de publication : pour éviter les phénomènes de fascination liés à la spectacularité et la ludicité des dispositifs numériques, il faut aussi enseigner le décryptage des stratégies de communication inscrites au cœur des matérialités des dispositifs numériques et interroger les rapports de pouvoir sous-jacents.

\section{BIBLIOGRAPHIE}

AKRICH, M. (2010). « Comment décrire les objets techniques?». Techniques et Culture 54-55, p. 205-219.

AUDET, R. (2015). « Le difficile art de la critique du livre numérique ». L'épée du soleil. Mis en ligne le 14 janvier 2015. En ligne : http://carnets.contemporain.info/audet/archives/1345. Consulté le 25 juin 2019.

BELISLE, C. (2006). « Le livre électronique : vers une lecture instrumentée ». In : Rouet J.-F., Germain B., Mazel I. (Éds), Lecture et technologies numériques, Enjeux et défis des technologies numériques pour l'enseignement et les pratiques de lecture. Paris : SCÉRÉN-CNDP, p. 49-66.

BOLTER, J. D. \& GRUSIN, R. (2000). Remédiation. Understanding New Media. Cambridge/Londres : MIT Press.

BOUCHARDON, S. (2014). La valeur heuristique de la littérature numérique. Paris : Hermann. 
BOUTIN, J.-F., LACELLE, N., \& LEBRUN, M. (2012). « La littératie médiatique multimodale : de nouvelles approches en lecture-écriture à l'école et hors de l'école ». Presses de l'Université du Québec.

BOUTIN, J.-F., LACELLE, N., \& LEBRUN, M. (2017). La littératie médiatique multimodale appliquée en contexte numérique.LMM@: Outils conceptuels et didactiques. Québec : Presses de l'Université du Québec.

Délégation académique au numérique pour l'éducation. Lycées 4.0. En ligne : https://www4.acnancy-metz.fr/dane/wp/lycee-4-0/.

Eduscol. Livre numérique. En ligne : https://eduscol.education.fr/numerique/dossier/lectures/ livrelec. Consulté le 25 juin 2019.

Eduscol. Le numérique comme vecteur de création. En ligne : https://eduscol.education.fr/lettres/ enseigner/ressources-et-usages-numeriques/pratiques-numeriques/reflechir-sur-le-numerique/ vecteur-de-creation.html. Consulté le 26 juin 2019.

GERVAIS, B. (1998). « Une lecture sans tradition : lire à la limite de ses habitudes ». Protée 25 (3), p. 7-20. En ligne : http://oic.uqam.ca/fr/publications/une-lecture-sans-tradition-lire-a-la-limitede-ses-habitudes. Consulté le 26 juin 2019.

GERVAIS, B. \& XANTHOS, N. (1999). «L'hypertexte : une lecture sans fin ». In : Vuillemin, A. \& Lenoble, M. (éds), Littérature, informatique, lecture. De la lecture assistée par ordinateur à la lecture interactive. Limoges : Presses Universitaires de Limoges, p. 111-125.

GOODY, J. \& MELOT, M. (2006). « La place du livre dans le monde de l'écrit. Entretien entre Jack Goody et Michel Melot ", Pratiques 131-132, p. 76-82.

GROSSMANN, F. (1996). «Que devient la littérature enfantine lorsqu'on la lit aux enfants d'école maternelle? ». In : Tauveron, C. \& Reuter, Y. (dirs). Repères 13, p. 85-101.

GUILET, A. (2015). « Les œuvres hypermédiatiques littéraires pour tablettes tactiles (1) ». Mis en ligne le 5 février 2015. En ligne : http://nt2.uqam.ca/fr/delineaires/les-oeuvreshypermediatiques-litteraires-pour-tablettes-tactiles-1. Consulté le 26 juin 2019.

HALL, S. (1994) [1973]. « Codage/décodage ». Réseaux 68, p. 27-39.

HAYLES, K. N. (2017) [2012]. Lire et penser en milieux numériques. Attention, récits, technogenèse. Trad. de l'anglais par C. Degoutin. Grenoble : Éditions littéraires et linguistiques de l'université de Grenoble.

JAJAH, M. (2010). « Le "livre enrichi" : définitions, précisions, mises au point (pas encore très au point) ». SoBookOnline. Mis en ligne le 28 novembre 2010. En ligne : https:// sobookonline.wordpress.com/2010/11/28/le-livre-enrichi-definitions-precisions-mises-au-pointpas-tres-au-point/. Consulté le 28 juin 2019.

JEANNERET, Y. (2014). Critique de la trivialité. Les médiations de la communication, enjeux de pouvoir. Paris : Éditions Non Standard.

JEANNERET, Y. (2007). « Les semblants du papier : l'investissement des objets comme travail de la mémoire sémiotique ». Communication et langages 153, p. 79-94.

JEANNERET, Y., \& SOUCHIER, E. (2005). « L'énonciation éditoriale dans les écrits d'écran ». Communication et langages 145, p. 3-15.

KAPLAN, F. (2012). « How books will become machines ». In : Clivaz, C., et al. (éds). Lire demain. Des manuscrits antiques à l'ère digitale. Lausanne : Presses polytechniques et universitaires romandes, p. 27-44. 
KRESS, G. (2010). Multimodality : a Social Semiotic Approach to Contemporary Communication. Londres/ New York : Routledge.

LABORDERIE, A., JEANTET, C., \& HELLEGOUARC'H, P. (2018). « Remédiatiser le patrimoine littéraire à travers le livre enrichi : quelles expériences de lecture ?». Revue de recherches en littératie médiatique multimodale, 8. En ligne : https://doi.org/10.7202/1050941ar

LANDOW, G. P. (1992). Hypertext. The Convergence of Contemporary Critical Theory and Technology. Baltimore : John Hopkins University Press.

Legifrance (2011). Décret $n^{\circ}$ 2011-1499 du 10 novembre 2011 pris en application de la loi $n^{\circ} 2011-590$ du 26 mai 2011 relative au prix du livre numérique - Article 1. En ligne : https://www.legifrance.gouv.fr/eli/ decret/2011/11/10/2011-1499/jo/article_1. Consulté le 26 juin 2019.

MANGEN, A. (2008). « Hypertext fiction reading: haptics and immersion ». Journal of Research in Reading 31 (4), p. 404-419.

Ministère de l'Éducation nationale et de la Jeunesse (2018a). Le numérique et les programmes actualisés. En ligne : https://eduscol.education.fr/cid133066/le-numerique-et-les-programmesactualises.html.

Ministère de l'Éducation nationale et de la Jeunesse (2018b). Le numérique et les programmes de cycle 4. En ligne : https://cache.media.eduscol.education.fr/file/CRCNum/91/9/

Reperes_progressivite_accessible_926919.pdf.

PINI, M. (2019). «L'album numérique, un nouveau pacte de lecture pour des enfants de maternelle ». Mémoire de master 2, dans le cadre de l'option numérique du parcours Innovation et développement personnel (mention PIF). Nancy : Institut National Supérieur du Professorat et de l'Éducation.

PRost, B., MAURIN, X., \& LeKeHAL, M. (2013). Le Livre numérique. Paris : Éditions du Cercle de la librairie.

RIO, F. (2014). « Le livre augmenté : pour une innovation technique et narrative ». Mémoires du livre/Studies in Book Culture 5 (2). En ligne : https://doi.org/10.7202/1024782ar

ROBERT, P. (2009). Une théorie sociétale des TIC. Penser les TIC entre approche critique et modélisation conceptuelle. Paris : Hermès-Lavoisier.

SAEMMER, A. (2015). Rhétorique du texte numérique. Villeurbanne : Presses de l'Enssib.

SAEMMER, A. (2011). « Lectures immersives du texte numérique - un paradoxe ? ». In : Bootz, P. \& Fenniche Daoues, R. Lire dans un monde numérique. Villeurbanne : Presses de l'Enssib, p. 255-276. SAEMMER, A. \& TREHONDART, N. (dirs) (2017). Livres d'art numériques. De la conception à la réception. Paris : Hermann.

TREHONDART, N. (2016). Le livre numérique enrichi : conception, modélisations de pratiques, réception. Thèse de doctorat en sciences de l'information et de la communication. Saint-Denis : Université de Paris 8 Vincennes-Saint-Denis.

VANDENDORPE, C. (1999). Du papyrus à l'hypertexte. Essai sur les mutations du texte et de la lecture. Paris : La Découverte.

VIDAL, G. (1998). «L'interactivité et les sites Web de musée ». Public et musées 13, p. 89-105. 


\section{NOTES}

1. Acronyme désignant les sociétés : Google, Amazon, Facebook, Apple.

2. Voir le dossier d'Eduscol consacré au livre numérique.

3. Voir la présentation de la Délégation académique au numérique pour l'éducation sur les lycées 4.0.

4. À la suite d'Y. Jeanneret (2014), nous adoptons ici une définition large du « texte » en l'étendant aux productions plurisémiotiques.

5. L'ePub est un format industriel qui hérite des standards du World Wide Web et nécessite un logiciel de lecture pour actualiser ses formes. Il diffère du livre-application qui, selon F. Kaplan (2012), « intègre la définition de son interactivité au sein de sa propre structure » et intègre son propre logiciel de lecture.

6. Legifrance, 2011.

7. Extrait des programmes du cycle 3 (Ministère de l'Éducation nationale et de la Jeunesse, 2018a).

8. Extrait des programmes du cycle 4 (Ministère de l'Éducation nationale et de la Jeunesse, 2018b).

9. La majorité des interviewés étaient des pure players de l'édition, publiant exclusivement au format numérique. 11 entretiens ont été menés avec des concepteurs de livres d'art numériques et 19 avec des concepteurs de fictions enrichies sur tablette. Liste non exhaustive des maisons d'édition numériques ayant accepté de participer à cette enquête: La Souris qui raconte, Byook, Actialuna, Les Inéditeurs, Studio Troll, L'Apprimerie, Art Book Magazine, Pandore Éditions, e-Toiles, Poésie Industrielle, La Dentellière... La plupart d'entre eux proviennent des secteurs de l'édition, du Web, de la musique, de l'audiovisuel ou encore du jeu vidéo. Seul deux responsables éditoriaux provenaient de maisons d'édition établies de longue date: la Réunion des musées nationaux et le centre George Pompidou. Nous les avons anonymisés en les numérotant de A1 à A33.

10. À la différence de la liseuse dédiée uniquement à la lecture de livres numériques, la tablette permet, outre la lecture de livres numériques, de regarder des films, d'écouter de la musique, de prendre des photographies, de naviguer sur le Web, de communiquer grâce à des applications sociales.

11. Le catalogue d'exposition numérique Edward Hopper, d'une fenêtre à l'autre a été édité et commercialisé en 2012 sous la forme d'une application pour iPad par la Réunion des musées nationaux, en lien avec l'exposition très médiatisée sur le peintre qui s'est tenue au Grand Palais en 2011-2012. Voir sa présentation sur le site de l'éditeur.: https://www.grandpalais.fr/fr/article/dune-fenetre-lautre-lapplication-hopper-pourlipad.

12. G. Vidal (1998, p. 92) définit l'interactivité comme la "possibilité technique pour l'usager de faire des combinaisons à travers l'hypertexte et l'hypermédia, offrant un certain choix dans la navigation à partir d'images, de sons et de textes pour définir un type d'accès ".

13. Voir Le numérique comme vecteur de création sur le site Eduscol. 


\section{RÉSUMÉS}

Convoquant une pluralité de ressources sémiotiques (texte, son, vidéo, images animées...), le livre numérique enrichi est devenu un support d'apprentissage régulièrement mobilisé dans les pratiques pédagogiques (manuels numériques, création d'ebooks sur tablettes tactiles). En prenant appui sur des entretiens que nous avons menés entre 2011 et 2016 auprès d'une trentaine de concepteurs de livres numériques, nous examinons dans cet article la manière dont ce dispositif symbolique et technique, à travers ses formes graphiques et les représentations souvent paradoxales qu'il suscite, pourrait constituer une porte d'entrée pertinente dans l'apprentissage d'une « littératie médiatique multimodale » en contexte numérique.

Convoking a plurality of semiotic resources (text, sound, video, animated images, etc.), the enhanced digital book has become a learning medium that is regularly used in teaching practices (digital textbooks, creation of ebooks on touch pads). Based on interviews we conducted between 2011 and 2016 with some thirty ebook designers, in this article we examines how this symbolic and technical device, through its graphic forms and the often paradoxical representations it generates, could constitute a relevant entry point for learning "multimodal media literacy" in a digital context.

\section{INDEX}

Mots-clés : livre numérique enrichi, littératie médiatique multimodale, lecture numérique, tablette numérique, pratiques d'édition

Keywords : enhanced ebook, multimodal media literacy, digital reading, reading device, publishing practices

\section{AUTEUR}

\section{NOLWENN TRÉHONDART}

Université de Lorraine, Crem, F-57000 Metz, France 